\title{
On the Meyniel condition for hamiltonicity in bipartite digraphs
}

\author{
Janusz Adamus ${ }^{12 \mid k} \quad$ Lech Adamus $\left.\right|^{3} \quad$ Anders Yeo ${ }^{45}$ \\ ${ }^{1}$ The University of Western Ontario, London, Ontario, Canada \\ ${ }^{2}$ Polish Academy of Sciences, Warsaw, Poland \\ ${ }^{3}$ AGH University of Science and Technology, Krakow, Poland \\ ${ }^{4}$ Singapore University of Technology and Design, Singapore \\ ${ }^{5}$ University of Johannesburg, South Africa \\ received 23 ${ }^{\text {rd }}$ Apr. 2013, revised $1^{\text {st }}$ Mar. 2014, accepted 25 $5^{\text {th }}$ Apr. 2014.
}

We prove a sharp Meyniel-type criterion for hamiltonicity of a balanced bipartite digraph: For $a \geq 2$, a strongly connected balanced bipartite digraph $D$ on $2 a$ vertices is hamiltonian if $d(u)+d(v) \geq 3 a$ whenever $u v \notin A(D)$ and $v u \notin A(D)$. As a consequence, we obtain a sharp sufficient condition for hamiltonicity in terms of the minimal degree: a strongly connected balanced bipartite digraph $D$ on $2 a$ vertices is hamiltonian if $\delta(D) \geq 3 a / 2$.

Keywords: digraph, bipartite digraph, cycle, cycle factor, hamiltonicity, degree condition

\section{Introduction}

\subsection{Results}

The main goal of this article is to prove a Meyniel-type sufficient condition for hamiltonicity of a balanced bipartite digraph. We consider digraphs in the sense of [3], and use standard graph theoretical terminology and notation (see Section 1.2 for details).

Our object of study in the present article is the class of bipartite digraphs satisfying the following Meyniel-type condition (cf. Thm.1.6).

Definition 1.1. Consider a balanced bipartite digraph $D$ on $2 a$ vertices. For $k \geq 0$, we will say that $D$ satisfies condition $\left(\mathcal{M}_{k}\right)$ when

$$
d(u)+d(v) \geq 3 a+k
$$

for every pair of distinct vertices $u, v \in V(D)$ such that $u v \notin A(D)$ and $v u \notin A(D)$.

Our main result is the following:

\footnotetext{
*Email: jadamus@uwo. ca. Research partially supported by Natural Sciences and Engineering Research Council of Canada.

$\dagger$ Email: adamus@agh. edu.pl. Research partially supported by Polish Ministry of Science and Higher Education.

‡Email: andersyeo@gmail.com 
Theorem 1.2. Let $D$ be a balanced bipartite digraph on 2 a vertices, where $a \geq 2$. Then $D$ is hamiltonian provided one of the following holds:

(a) D satisfies condition $\left(\mathcal{M}_{1}\right)$, or

(b) D is strongly connected and satisfies condition $\left(\mathcal{M}_{0}\right)$.

(A digraph is called strongly connected when, for every (ordered) pair of distinct vertices $u$ and $v, D$ contains a directed path originating in $u$ and terminating in $v$.)

There are numerous sufficient conditions for existence of hamiltonian cycles in digraphs (see [3, 5]). In this article, we will be concerned with the degree conditions. For general digraphs, let us recall the following four classical results.

Theorem 1.3 (Ghouila-Houri, 1960, [7]). Let D be a strongly connected digraph on $n$ vertices, where $n \geq 3$. If $\delta(D) \geq n$, then $D$ is hamiltonian.

Theorem 1.4 (Nash-Williams, 1969, [10]). Let $D$ be a digraph on $n$ vertices, where $n \geq 3$. If $\delta^{+}(D) \geq$ $n / 2$ and $\delta^{-}(D) \geq n / 2$, then $D$ is hamiltonian.

Theorem 1.5 (Woodall, 1972, [11]). Let $D$ be a digraph on $n$ vertices, where $n \geq 3$. If $d^{+}(u)+d^{-}(v) \geq$ $n$ for every pair of distinct vertices $u, v \in V(D)$ satisfying $u v \notin A(D)$, then $D$ is hamiltonian.

Theorem 1.6 (Meyniel, 1973, [9]). Let $D$ be a strongly connected digraph on $n$ vertices, where $n \geq 3$. If $d(u)+d(v) \geq 2 n-1$ for any two vertices $u$ and $v$ such that $u v \notin A(D)$ and $v u \notin A(D)$, then $D$ is hamiltonian.

All the above criteria are sharp (see [5]). Note also that Theorems 1.3, 1.4 and 1.5 follow from Theorem 1.6. A beautiful short proof of the latter can be found in [6].

Naturally, for bipartite digraphs one might expect bounds for degrees of order $|D| / 2$ rather than $|D|$. This is the case, indeed, for analogues of the Nash-Williams and Woodall theorems. As for the analogues of the Ghouila-Houri and Meyniel theorems, however, this expectation is quite far from reality (cf. Remark 1.10]. For minimal half-degrees we have the following result.

Theorem 1.7 (Amar \& Manoussakis, 1990, [2]). Let D be a balanced bipartite digraph on $2 a$ vertices, where $a \geq 2$. If $\delta^{+}(D) \geq(a+2) / 2$ and $\delta^{-}(D) \geq(a+2) / 2$, then $D$ is hamiltonian.

The above criterion is sharp. Moreover, it is shown in [2] that the only non-hamiltonian digraph $D$ satisfying $\delta^{+}(D) \geq(a+1) / 2$ and $\delta^{-}(D) \geq(a+1) / 2$ is the digraph on 6 vertices depicted in Fig. 1 .

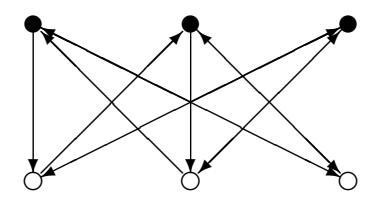

Fig. 1

An analogue of Woodall's theorem was given by Manoussakis and Millis in [8], and recently considerably strengthened by J. Adamus and L. Adamus. 
Theorem 1.8 (Adamus \& Adamus, 2012, [1]). Let $D$ be a balanced bipartite digraph on $2 a$ vertices, where $a \geq 2$. If $d^{+}(u)+d^{-}(v) \geq a+2$ for every pair of vertices $u$ and $v$ from the opposite colour classes such that uv $\notin A(D)$, then $D$ is hamiltonian.

In the present paper, we give bipartite analogues of the Ghouila-Houri and Meyniel theorems. These are Theorems 1.9 (below) and 1.2, respectively. Quite surprisingly, the bounds on degrees are much bigger than one might expect from Theorems 1.7 and 1.8 above. It is worth remarking that the methods of [6] do not carry over to the bipartite case.

Theorem 1.9. Let $D$ be a balanced bipartite digraph on 2 a vertices, where $a \geq 2$. Then $D$ is hamiltonian provided one of the following holds:

(a) $\delta(D) \geq(3 a+1) / 2$, or

(b) $D$ is strongly connected and $\delta(D) \geq 3 a / 2$.

Of course, Theorem 1.9 is an immediate corollary of Theorem 1.2 .

Remark 1.10. The bounds in Theorems 1.2 and 1.9 are sharp, as can be seen in the following two examples. Example 1.11 provides, for every even $a \geq 2$, a non-hamiltonian balanced bipartite digraph $D(a)$ on $2 a$ vertices in which every vertex has degree $3 a / 2$. The second example (due to Amar and Manoussakis [2]) shows that, for every $a \geq 3$ and every $1 \leq l<a / 2$, there is a non-hamiltonian strongly connected balanced bipartite digraph $D(a, l)$ on $2 a$ vertices with $\delta(D(a, l))=a+l$.

Example 1.11. Let $a$ be a positive even integer, and let $D(a)$ be a bipartite digraph with colour classes $V_{1}$ and $V_{2}$ such that $V_{1}$ (resp. $V_{2}$ ) is a disjoint union of sets $R, S$ (resp. $U, W$ ) of cardinality $a / 2$ each, and $A(D(a))$ consists of the following arcs:

(a) $r y$, for all $r \in R$ and $y \in V_{2}$,

(b) $u x$, for all $u \in U$ and $x \in V_{1}$, and

(c) $s w$ and $w s$, for all $s \in S$ and $w \in W$.

Then every vertex of $D(a)$ is of degree $3 a / 2$, but $D(a)$ contains no hamiltonian cycle. Notice that $D(a)$ is not strongly connected.

Example 1.12. For $a \geq 3$ and $1 \leq l<a / 2$, let $D(a, l)$ be a bipartite digraph with colour classes $V_{1}$ and $V_{2}$ such that $V_{1}$ (resp. $V_{2}$ ) is a disjoint union of sets $R, S$ (resp. $U, W$ ) with $|R|=|U|=l$, $|S|=|W|=a-l$, and $A(D(a, l))$ consists of the following arcs:

(a) $r y$ and $y r$, for all $r \in R$ and $y \in V_{2}$,

(b) $u x$ and $x u$, for all $u \in U$ and $x \in V_{1}$, and

(c) $s w$, for all $s \in S$ and $w \in W$.

Then $\delta(D(a, l))=a+l$. In particular, for odd $a, \delta(D(a,(a-1) / 2))=(3 a-1) / 2$. Notice that $D(a, l)$ is strongly connected, but not hamiltonian.

Remark 1.13. It is also interesting to observe that under the assumptions (a) in Theorems 1.2 and 1.9 , the strong-connectedness is redundant. In fact, condition $\left(\mathcal{M}_{1}\right)$ implies a much stronger property: a bipartite digraph $D$ satisfying condition $\left(\mathcal{M}_{1}\right)$ contains a perfect matching $M$ (cf. Lemma 2.1), and, for every pair of distinct vertices $u, v, D$ contains a directed path from $u$ to $v$ which is compatible with $M$ (i.e., a path whose every other arc belongs to $M$; cf. Lemma 2.2.

Finally, notice that conditions $\left(\mathcal{M}_{k}\right)$ cannot be weakened to apply only to pairs of vertices from the opposite colour classes (à la Theorem 1.8). This follows from the fact that there exist strongly connected 
non-hamiltonian balanced bipartite tournaments (Example 1.14 below). Recall that a bipartite tournament is a bipartite digraph $D$ in which, for every pair of vertices $x, y$ from the opposite colour classes, precisely one of the arcs $x y, y x$ belongs to $A(D)$.

Example 1.14. For $a \geq 3$ and $1 \leq l<a / 2$, let $T(a, l)$ be a bipartite digraph with colour classes $V_{1}$ and $V_{2}$ such that $V_{1}$ (resp. $V_{2}$ ) is a disjoint union of sets $R, S$ (resp. $U, W$ ) with $|R|=|U|=l$, $|S|=|W|=a-l$, and $A(T(a, l))$ consists of the following arcs:

(a) $r u$, for all $r \in R$ and $u \in U$,

(b) $u s$, for all $u \in U$ and $s \in S$,

(c) $s w$, for all $s \in S$ and $w \in W$, and

(d) $w r$, for all $w \in W$ and $r \in R$.

Then $T(a, l)$ is strongly connected and vacuously satisfies condition $\left(\mathcal{M}_{1}\right)$ (hence also condition $\left(\mathcal{M}_{0}\right)$ ) for every pair of vertices from the opposite colour classes, but $T(a, l)$ contains no hamiltonian cycle.

\subsection{Notation and terminology}

A digraph $D$ is a pair $(V(D), A(D))$, where $V(D)$ is a finite set (of vertices) and $A(D)$ is a set of ordered pairs of distinct elements of $V(D)$, called $\operatorname{arcs}$ (i.e., $D$ has no loops or multiple arcs). The number of vertices $|V(D)|$ is the order of $D$ (also denoted by $|D|$ ). For vertices $u$ and $v$ from $V(D)$, we write $u v \in A(D)$ to say that $A(D)$ contains the ordered pair $(u, v)$.

For a vertex set $S \subset V(D)$, we denote by $N^{+}(S)$ the set of vertices in $V(D)$ dominated by the vertices of $S$; i.e.,

$$
N^{+}(S)=\{u \in V(D): v u \in A(D) \text { for some } v \in S\} .
$$

Similarly, $N^{-}(S)$ denotes the set of vertices of $V(D)$ dominating vertices of $S$; i.e,

$$
N^{-}(S)=\{u \in V(D): u v \in A(D) \text { for some } v \in S\} .
$$

If $S=\{v\}$ is a single vertex, the cardinality of $N^{+}(\{v\})$ (resp. $N^{-}(\{v\})$ ), denoted by $d^{+}(v)$ (resp. $d^{-}(v)$ ) is called the outdegree (resp. indegree) of $v$ in $D$. The degree of $v$ is $d(v)=d^{+}(v)+d^{-}(v)$. Further, by $\delta^{+}(D)$ and $\delta^{-}(D)$ we will denote respectively the minimal outdegree and the minimal indegree of $D$; i.e., $\delta^{+}(D)=\min \left\{d^{+}(v): v \in V(D)\right\}$ and $\delta^{-}(D)=\min \left\{d^{-}(v): v \in V(D)\right\}$. The minimal degree of $D$ will be denoted by $\delta(D)$.

For vertex sets $S, T \subset V(D)$, we denote by $A[S, T]$ the set of all arcs of $A(D)$ from a vertex in $S$ to a vertex in $T$. Let $\stackrel{\leftrightarrow}{a}(S, T)=|A[S, T]|+|A[T, S]|$. Note that $\stackrel{\leftrightarrow}{a}(\{v\}, V(D) \backslash\{v\})=d(v)$. A set of vertices $\left\{v_{1}, \ldots, v_{k}\right\}$ such that $\overleftrightarrow{a}\left(\left\{v_{i}\right\},\left\{v_{j}\right\}\right)=0$, for all $i \neq j$, is called independent.

A directed cycle (resp. directed path) on vertices $v_{1}, \ldots, v_{m}$ in $D$ is denoted by $\left[v_{1}, \ldots, v_{m}\right]$ (resp. $\left(v_{1}, \ldots, v_{m}\right)$ ). We will refer to them as simply cycles and paths (skipping the term "directed"), since their non-directed counterparts are not considered in this article at all.

A cycle passing through all the vertices of $D$ is called hamiltonian. A digraph containing a hamiltonian cycle is called a hamiltonian digraph. A cycle factor in $D$ is a collection of vertex-disjoint cycles $C_{1}, \ldots, C_{l}$ such that $V\left(C_{1}\right) \cup \cdots \cup V\left(C_{l}\right)=V(D)$.

A digraph $D$ is bipartite when $V(D)$ is a disjoint union of independent sets $V_{1}$ and $V_{2}$ (the colour classes). It is called balanced if $\left|V_{1}\right|=\left|V_{2}\right|$. One says that a bipartite digraph $D$ is complete when $d(x)=2\left|V_{2}\right|$ for all $x \in V_{1}$. 
A matching from $V_{1}$ to $V_{2}$ is an independent set of arcs with origin in $V_{1}$ and terminus in $V_{2}\left(u_{1} u_{2}\right.$ and $v_{1} v_{2}$ are independent arcs when $u_{1} \neq v_{1}$ and $u_{2} \neq v_{2}$ ). If $D$ is balanced, one says that such a matching is perfect if it consists of precisely $\left|V_{1}\right|$ arcs. After [2], we call a path compatible with a matching $M$ from $V_{1}$ to $V_{2}$ (or, $M$-compatible, for short) if its arcs are alternately in $M$ and in $A(D) \backslash M$.

For a perfect matching $M$ from $V_{1}$ to $V_{2}$ and a vertex $x^{\prime} \in V_{1}$, we will denote by $M\left(x^{\prime}\right)$ the unique vertex $y^{\prime} \in V_{2}$ such that $x^{\prime} y^{\prime} \in M$. Similarly, for $y^{\prime} \in V_{2}$, we will denote by $M^{-1}\left(y^{\prime}\right)$ the unique vertex $x^{\prime} \in V_{1}$ for which $x^{\prime} y^{\prime} \in M$. Finally, for a subset $S \subset V_{2}$, we will denote by $M^{-1}(S)$ the set $\left\{M^{-1}(y): y \in S\right\}$.

\section{Lemmas}

We prove Theorem 1.2 in Section 3 . Our argument is based on the following lemma.

Lemma 2.1. Let $D$ be a balanced bipartite digraph on $2 a$ vertices, where a $\geq 2$. Suppose that $D$ satisfies condition $\left(\mathcal{M}_{1}\right)$, or $D$ is strongly connected and satisfies condition $\left(\mathcal{M}_{0}\right)$. Then $D$ contains a cycle factor.

Proof: Let $V_{1}$ and $V_{2}$ denote the two colour classes of $D$. Observe that $D$ contains a cycle factor if and only if there exist both a perfect matching from $V_{1}$ to $V_{2}$ and a perfect matching from $V_{2}$ to $V_{1}$. Therefore, by the König-Hall theorem (see, e.g., [4]), it suffices to show that $\left|N^{+}(S)\right| \geq|S|$ for every $S \subset V_{1}$ and $\left|N^{+}(T)\right| \geq|T|$ for every $T \subset V_{2}$.

For a proof by contradiction, suppose that a non-empty set $S \subset V_{1}$ is such that $\left|N^{+}(S)\right|<|S|$. Then $V_{2} \backslash N^{+}(S) \neq \varnothing$ and, for every $y \in V_{2} \backslash N^{+}(S)$, we have $d^{-}(y) \leq a-|S|$, hence $d(y) \leq 2 a-|S|$. We now consider the following two cases.

\section{Case 1.}

$|S|>a / 2$.

Then $d(y)<2 a-a / 2=3 a / 2$ for all $y \in V_{2} \backslash N^{+}(S)$. Therefore, if $V_{2} \backslash N^{+}(S)$ contains at least two elements, say $y_{1}$ and $y_{2}$, then $d\left(y_{1}\right)+d\left(y_{2}\right)<3 a$, which contradicts condition $\left(\mathcal{M}_{0}\right)$ (hence also $\left(\mathcal{M}_{1}\right)$ ). Suppose then that $V_{2} \backslash N^{+}(S)=\{y\}$. This can only happen when $|S|=a$, that is, when $S=V_{1}$. In this case, however, the vertex $y$ is not dominated by any vertex of $D$. Clearly, such a digraph is not strongly connected. Without the strong connectedness assumption, in turn, we have $d(y)=d^{+}(y)+d^{-}(y) \leq a+0$, hence $d(y)+d\left(y^{\prime}\right) \leq a+2 a$, for any $y^{\prime} \in V_{2} \backslash\{y\}$, which contradicts condition $\left(\mathcal{M}_{1}\right)$.

\section{Case 2.}

$|S| \leq a / 2$.

If this is so then, for every $x \in S$, we have $d(x)=d^{-}(x)+d^{+}(x) \leq a+(|S|-1) \leq(3 a-2) / 2$. Therefore, if $S$ contains at least two elements, say $x_{1}$ and $x_{2}$, we get $d\left(x_{1}\right)+d\left(x_{2}\right) \leq 3 a-2$, which contradicts $\left(\mathcal{M}_{0}\right)$ (hence also $\left(\mathcal{M}_{1}\right)$ ). Suppose then that $S=\{x\}$, and hence $N^{+}(S)=\varnothing$, by our hypothesis. It follows that $x$ does not dominate any vertex in $D$, which leads to a contradiction if $D$ is assumed strongly connected. Without the strong connectedness assumption, in turn, we have $d(x)=d^{-}(x)+d^{+}(x) \leq a+0$, hence $d(x)+d\left(x^{\prime}\right) \leq a+2 a$, for any $x^{\prime} \in V_{1} \backslash\{x\}$, which contradicts condition $\left(\mathcal{M}_{1}\right)$.

This completes the proof of existence of a perfect matching from $V_{1}$ to $V_{2}$. The proof for a matching in the opposite direction is analogous. 
As advertised in Remark 1.13 every digraph $D$ satisfying condition $\left(\mathcal{M}_{1}\right)$ is strongly connected. In fact, much more is true: By Lemma 2.1 condition $\left(\mathcal{M}_{1}\right)$ implies that $D$ contains a perfect matching from $V_{1}$ to $V_{2}$. Moreover, $D$ contains a perfect matching $M$ such that any two vertices in $D$ can be connected by an $M$-compatible path. This is clear when $D$ is hamiltonian. The non-hamiltonian case is settled in the following lemma. (Note that the lemma is not needed for the proof of Theorem 1.2 .)

Lemma 2.2. Let $D$ be a balanced bipartite digraph on 2 a vertices, where a $\geq 2$, which satisfies condition $\left(\mathcal{M}_{1}\right)$. Let $V_{1}$ and $V_{2}$ be the colour classes of $D$, and let $M$ be a perfect matching from $V_{1}$ to $V_{2}$. Suppose that $D$ is not hamiltonian. Then, for every pair of distinct vertices $u, v \in V(D), D$ contains an $M$ compatible path from $u$ to $v$.

Proof: First, we claim that it suffices to show that $D$ contains an $M$-compatible path from $y$ to $x$ for every pair of vertices such that $y \in V_{2}$ and $x \in V_{1}$. Indeed, to find an $M$-compatible path in $D$ from $x^{\prime} \in V_{1}$ to $x^{\prime \prime} \in V_{1}$, it suffices to find an $M$-compatible path from $M\left(x^{\prime}\right)$ to $x^{\prime \prime}$. Likewise, to find an $M$-compatible path from $y^{\prime} \in V_{2}$ to $y^{\prime \prime} \in V_{2}$, it suffices to find an $M$-compatible path from $y^{\prime}$ to $M^{-1}\left(y^{\prime \prime}\right)$. Finally, to find an $M$-compatible path from $x^{\prime} \in V_{1}$ to $y^{\prime \prime} \in V_{2}$, it suffices to find an $M$-compatible path from $M\left(x^{\prime}\right)$ to $M^{-1}\left(y^{\prime \prime}\right)$ (unless $x^{\prime} y^{\prime \prime}$ already is in $M$ ).

Next, observe that one can assume

$$
d^{+}(v) \geq 2 \quad \text { and } \quad d^{-}(v) \geq 2 \quad \text { for all } v \in V(D) .
$$

Indeed, for if $d^{+}\left(v^{\prime}\right)<2$ for some $v^{\prime} \in V(D)$, then $d\left(v^{\prime}\right) \leq a+1$, hence, by condition $\left(\mathcal{M}_{1}\right), d(v) \geq 2 a$ for all $v \neq v^{\prime}$ from the same colour class. Since every degree is bounded above by $2 a$, we would actually have $d(v)=2 a$ for all $v \neq v^{\prime}$ from the colour class of $v^{\prime}$, as well as $d^{+}\left(v^{\prime}\right)=1$ and $d^{-}\left(v^{\prime}\right)=a$. It is readily seen that then $D$ would contain a hamiltonian cycle, contrary to our assumption. The argument for $d^{-}(v)$ is analogous.

Now, for a proof by contradiction, suppose that $y \in V_{2}$ and $x \in V_{1}$ are such that $D$ contains no path from $y$ to $x$ compatible with $M$. Let $V_{2}^{y}$ denote the set of vertices in $V_{2}$ which can be reached from $y$ by a path compatible with $M$, and let $V_{1}^{\text {no }}$ denote the set of vertices in $V_{1}$ which cannot be reached from $y$ by a path compatible with $M$. Then $y \in V_{2}^{y}, x \in V_{1}^{n o}$, and $A\left[V_{2}^{y}, V_{1}^{n o}\right]=\varnothing$, by the definition of $V_{2}^{y}$ and $V_{1}^{n o}$.

Note that, if $y^{\prime} \in V_{2} \backslash V_{2}^{y}$, then $M^{-1}\left(y^{\prime}\right) \in V_{1}^{n o}$, for otherwise there would be an $M$-compatible path from $y$ to $M^{-1}\left(y^{\prime}\right)$, hence also to $y^{\prime}$; a contradiction. This implies that $\left|V_{1}^{n o}\right| \geq\left|V_{2} \backslash V_{2}^{y}\right|=a-\left|V_{2}^{y}\right|$, and so $\left|V_{1}^{n o}\right|+\left|V_{2}^{y}\right| \geq a$. As $A\left[V_{2}^{y}, V_{1}^{n o}\right]=\varnothing$, it follows from 2.1) that $\left|V_{2}^{y}\right| \leq a-2$ and $\left|V_{1}^{n o}\right| \leq a-2$, which in turn implies that $\left|V_{2}^{y}\right| \geq 2$ and $\left|V_{1}^{n o}\right| \geq 2$ (as $\left|V_{1}^{n o}\right|+\left|V_{2}^{y}\right| \geq a$ ). Let then $u_{1}, v_{1} \in V_{1}^{n o}$ and $u_{2}, v_{2} \in V_{2}^{y}$ be four arbitrary vertices. By the above estimates, one gets that

$$
\begin{aligned}
\left(d\left(u_{1}\right)+d\left(v_{1}\right)\right)+\left(d\left(u_{2}\right)+d\left(v_{2}\right)\right) \leq 2\left(2 a-\left|V_{2}^{y}\right|\right)+2\left(2 a-\left|V_{1}^{n o}\right|\right) & \\
& =8 a-2\left(\left|V_{2}^{y}\right|+\left|V_{1}^{n o}\right|\right) \leq 8 a-2 a .
\end{aligned}
$$

Therefore, $d\left(u_{1}\right)+d\left(v_{1}\right) \leq 3 a$ or $d\left(u_{2}\right)+d\left(v_{2}\right) \leq 3 a$; a contradiction. 


\section{Proof of the main result}

\section{Proof of Theorem 1.2}

Let $D$ be a balanced bipartite digraph on $2 a$ vertices, and let $V_{1}$ and $V_{2}$ denote its colour classes. We will proceed by induction on $a$. For $a=2$ the theorem is clearly true, so suppose that $a \geq 3$ and the theorem holds for $a-1$.

By Lemma 2.1. $D$ contains a cycle factor $C_{1}, C_{2}, \ldots, C_{l}$. Assume that $l$ is minimum possible, and for a proof by contradiction suppose that $l \geq 2$. Recall that $\left|C_{i}\right|$ denotes the order of cycle $C_{i}$. Without loss of generality, assume that $\left|C_{1}\right| \leq\left|C_{2}\right| \leq \cdots \leq\left|C_{l}\right|$.

\section{Claim 1:}

$\overleftrightarrow{a}\left(V\left(C_{i}\right), V\left(C_{j}\right)\right) \leq \frac{\left|C_{i}\right| \cdot\left|C_{j}\right|}{2}$, for all $i \neq j$.

\section{Proof of Claim 1.}

Let $q \in\{1,2\}, x_{i} \in V\left(C_{i}\right) \cap V_{q}$ and $x_{j} \in V\left(C_{j}\right) \cap V_{q}$ be arbitrary. Let $x_{i}^{+}$be the successor of $x_{i}$ in $C_{i}$ and let $x_{j}^{+}$be the successor of $x_{j}$ in $C_{j}$. Let $\mathcal{Z}_{q}\left(x_{i}, x_{j}\right)$ be defined as $A(D) \cap\left\{x_{i} x_{j}^{+}, x_{j} x_{i}^{+}\right\}$. If $\left|\mathcal{Z}_{q}\left(x_{i}, x_{j}\right)\right|=2$ for some $x_{i}, x_{j}$, then the cycles $C_{i}$ and $C_{j}$ can be merged into one cycle by deleting the $\operatorname{arcs} x_{i} x_{i}^{+}$and $x_{j} x_{j}^{+}$and adding the $\operatorname{arcs} x_{i} x_{j}^{+}$and $x_{j} x_{i}^{+}$. This would contradict the minimality of $l$, so we may assume that

$$
\left|\mathcal{Z}_{q}\left(x_{i}, x_{j}\right)\right| \leq 1 \quad \text { for all } x_{i} \in V\left(C_{i}\right) \cap V_{q} \text { and } x_{j} \in V\left(C_{j}\right) \cap V_{q} \text {. }
$$

Now, consider an arc $u v \in A\left[V\left(C_{i}\right), V\left(C_{j}\right)\right]$ and assume $u \in V_{q}$. Let $v^{-}$denote the predecessor of $v$ in $C_{j}$. Then $u v \in \mathcal{Z}_{q}\left(u, v^{-}\right)$. Similarly, if $u v \in A\left[V\left(C_{j}\right), V\left(C_{i}\right)\right], u \in V_{q}$, and $v^{-}$is the predecessor of $v$ in $C_{i}$, then $u v \in \mathcal{Z}_{q}\left(v^{-}, u\right)$. Therefore

$$
\overleftrightarrow{a}\left(V\left(C_{i}\right), V\left(C_{j}\right)\right) \leq \sum_{q=1}^{2} \sum_{x_{i} \in V\left(C_{i}\right) \cap V_{q}} \sum_{x_{j} \in V\left(C_{j}\right) \cap V_{q}}\left|\mathcal{Z}_{q}\left(x_{i}, x_{j}\right)\right|
$$

and hence, by (3.1),

$$
\overleftrightarrow{a}\left(V\left(C_{i}\right), V\left(C_{j}\right)\right) \leq 2 \cdot \frac{\left|C_{i}\right|}{2} \cdot \frac{\left|C_{j}\right|}{2},
$$

which completes the proof of Claim 1.

We now return to the proof of Theorem 1.2. Repeatedly using Claim 1, we note that the following holds

$$
\begin{aligned}
\overleftrightarrow{a}\left(V\left(C_{1}\right) \cap V_{1}, V(D) \backslash V\left(C_{1}\right)\right)+\overleftrightarrow{a}\left(V\left(C_{1}\right) \cap V_{2}, V(D) \backslash V\left(C_{1}\right)\right) \\
=\overleftrightarrow{a}\left(V\left(C_{1}\right), V(D) \backslash V\left(C_{1}\right)\right)=\sum_{j=2}^{l} \stackrel{\leftrightarrow}{a}\left(V\left(C_{1}\right), V\left(C_{j}\right)\right) \leq \frac{\left|C_{1}\right|\left(2 a-\left|C_{1}\right|\right)}{2}
\end{aligned}
$$

Without loss of generality, we may assume that

$$
\overleftrightarrow{a}\left(V\left(C_{1}\right) \cap V_{1}, V(D) \backslash V\left(C_{1}\right)\right) \leq \frac{\left|C_{1}\right|\left(2 a-\left|C_{1}\right|\right)}{4},
$$


as otherwise

$$
\stackrel{\leftrightarrow}{a}\left(V\left(C_{1}\right) \cap V_{2}, V(D) \backslash V\left(C_{1}\right)\right) \leq \frac{\left|C_{1}\right|\left(2 a-\left|C_{1}\right|\right)}{4} .
$$

In other words, the average number of arcs between a vertex in $V\left(C_{1}\right) \cap V_{1}$ and $V(D) \backslash V\left(C_{1}\right)$ is bounded above by $\left(2 a-\left|C_{1}\right|\right) / 2$ (as $\left.\left|V\left(C_{1}\right) \cap V_{1}\right|=\left|C_{1}\right| / 2\right)$. We now consider the following two cases.

\section{Case 1.}

$\left|C_{1}\right| \geq 4$.

Let $x_{1}, x_{2} \in V\left(C_{1}\right) \cap V_{1}$ be distinct and chosen so that $\stackrel{\leftrightarrow}{a}\left(\left\{x_{1}, x_{2}\right\}, V(D) \backslash V\left(C_{1}\right)\right)$ is minimum. By the above formula we note that $\stackrel{\leftrightarrow}{a}\left(\left\{x_{1}, x_{2}\right\}, V(D) \backslash V\left(C_{1}\right)\right) \leq 2 a-\left|C_{1}\right|$. Since any vertex in $C_{1}$ has at most $\left|C_{1}\right|$ arcs to other vertices in $C_{1}$ (as there are $\left|C_{1}\right| / 2$ vertices from $V_{2}$ in $C_{1}$ ) and $\left|C_{1}\right| \leq a$, we get that

$$
d\left(x_{1}\right)+d\left(x_{2}\right) \leq 2\left|C_{1}\right|+2 a-\left|C_{1}\right|=2 a+\left|C_{1}\right| \leq 3 a,
$$

which leads to contradiction if $D$ is assumed to satisfy condition $\left(\mathcal{M}_{1}\right)$.

Suppose then that $D$ is strongly connected and satisfies condition $\left(\mathcal{M}_{0}\right)$, and we have equality in 3.4. It follows that there must be equalities in all the estimates that led to (3.4) as well. That is,

$$
\begin{gathered}
\overleftrightarrow{a}\left(\left\{x_{1}, x_{2}\right\}, V(D) \backslash V\left(C_{1}\right)\right)=2 a-\left|C_{1}\right|, \\
\stackrel{\leftrightarrow}{a}\left(\left\{x_{1}\right\}, V\left(C_{1}\right)\right)=\stackrel{\leftrightarrow}{a}\left(\left\{x_{2}\right\}, V\left(C_{1}\right)\right)=\left|C_{1}\right|, \\
\left|C_{1}\right|=a .
\end{gathered}
$$

By the choice of $x_{1}$ and $x_{2}$, it now follows from 3.3) and 3.5 that $\stackrel{\leftrightarrow}{a}\left(\left\{x_{i}, x_{j}\right\}, V(D) \backslash V\left(C_{1}\right)\right)=$ $2 a-\left|C_{1}\right|$ for any distinct $x_{i}, x_{j} \in V\left(C_{1}\right) \cap V_{1}$. Therefore, either we can find a pair of vertices $x_{1}^{\prime}, x_{2}^{\prime} \in$ $V\left(C_{1}\right) \cap V_{1}$ with $d\left(x_{1}^{\prime}\right)+d\left(x_{2}^{\prime}\right)<3 a$, or else condition 3.6) holds for all vertices from $V\left(C_{1}\right) \cap V_{1}$. The former would contradict condition $\left(\mathcal{M}_{0}\right)$, so we get that $\stackrel{\leftrightarrow}{a}\left(\{x\}, V\left(C_{1}\right)\right)=\left|C_{1}\right|$ for every $x \in$ $V\left(C_{1}\right) \cap V_{1}$. In other words, $D$ contains a complete bipartite digraph spanned on the vertices of $C_{1}$.

Next observe that, by minimality of $\left|C_{1}\right|$, (3.7) implies that $l=2$ and $\left|C_{1}\right|=\left|C_{2}\right|=a$. Consequently, we can swap $C_{1}$ and $C_{2}$ and repeat the above argument to get that $D$ contains also a complete bipartite digraph spanned on the vertices of $C_{2}$.

Now, we claim that

(i) $A\left[V\left(C_{1}\right) \cap V_{1}, V\left(C_{2}\right)\right] \neq \varnothing$ and $A\left[V\left(C_{2}\right), V\left(C_{1}\right) \cap V_{2}\right] \neq \varnothing$, or

(ii) $A\left[V\left(C_{1}\right) \cap V_{2}, V\left(C_{2}\right)\right] \neq \varnothing$ and $A\left[V\left(C_{2}\right), V\left(C_{1}\right) \cap V_{1}\right] \neq \varnothing$.

Indeed, condition $\left(\mathcal{M}_{0}\right)$ applied to pairs of vertices from $V\left(C_{1}\right) \cap V_{1}$ implies that there exists $x \in V\left(C_{1}\right) \cap$ $V_{1}$ with $\stackrel{\leftrightarrow}{a}\left(\{x\}, V\left(C_{2}\right)\right)>0$. Similarly, there exists $y \in V\left(C_{1}\right) \cap V_{2}$ such that $\stackrel{\leftrightarrow}{a}\left(\{y\}, V\left(C_{2}\right)\right)>0$. Therefore, if neither (i) nor (ii) held, then all the arcs between $C_{1}$ and $C_{2}$ would need to go in the same direction (i.e., either $A\left[V\left(C_{1}\right), V\left(C_{2}\right)\right]=\varnothing$ or $A\left[V\left(C_{2}\right), V\left(C_{1}\right)\right]=\varnothing$ ). But such an arrangement is impossible in a strongly connected digraph.

Thus, without loss of generality we can assume that $D$ contains an arc from $V\left(C_{1}\right) \cap V_{1}$ to $V\left(C_{2}\right)$ and an arc from $V\left(C_{2}\right)$ to $V\left(C_{1}\right) \cap V_{2}$. Then, however, $D$ must be hamiltonian, because it contains complete bipartite digraphs on $V\left(C_{1}\right)$ and on $V\left(C_{2}\right)$. This contradiction completes the proof of Case 1. 


\section{Case 2.}

$\left|C_{1}\right|<4$.

In this case $\left|C_{1}\right|=2$. Let $V\left(C_{1}\right) \cap V_{1}=\{x\}$ and $V\left(C_{1}\right) \cap V_{2}=\{y\}$. Note that, by (3.3), we have $d(x) \leq 2+\left(2 a-\left|C_{1}\right|\right) / 2=a+1$. Hence, if $D$ satisfies condition $\left(\mathcal{M}_{1}\right)$, then we can assume that $d\left(x^{\prime}\right)=2 a$ for all $x^{\prime} \in V_{1} \backslash\{x\}$. If $x u \in A(D)$ and $u \in C_{j}$ with $j>1$, then let $u^{-}$be the predecessor of $u$ in $C_{j}$ and note that we can merge $C_{1}$ and $C_{j}$ into one cycle using the arcs $x u$ and $u^{-} y$ (which exists as $d\left(u^{-}\right)=2 a$ ) instead of $x y$ and $u^{-} u$, which contradicts the minimality of $l$. Therefore there are no arcs from $x$ to $V(D) \backslash\{y\}$. Analogously, there are no arcs $u x$ from $V(D) \backslash\{y\}$ to $x$ (as we could then merge using $u x$ and $y u^{+}$, where $u^{+}$is the successor of $u$ on its cycle). Hence $d(x)=2$, and so $d(x)+d\left(x^{\prime}\right)=2 a+2<3 a+1$ for every $x^{\prime} \in V_{1} \backslash\{x\}$, which contradicts condition $\left(\mathcal{M}_{1}\right)$.

Suppose then that $D$ is strongly connected and satisfies condition $\left(\mathcal{M}_{0}\right)$. We can then assume that

$$
d\left(x^{\prime}\right) \geq 2 a-1, \text { for all } x^{\prime} \in V_{1} \backslash\{x\} .
$$

Hence, for all such $x^{\prime}, A(D)$ contains at least one of the arcs $x^{\prime} y$ and $y x^{\prime}$. The remainder of the proof is divided into two subcases depending on the actual value of $d(x)$.

\section{Case $2 a$.}

$d(x)=a+1$.

Then, by $[3.2), d(y) \leq a+1$ and hence, as above,

$$
d\left(y^{\prime}\right) \geq 2 a-1, \text { for all } y^{\prime} \in V_{2} \backslash\{y\} .
$$

We now want to show that $l=2$. Let $D^{\prime}$ be the sub-digraph of $D$ spanned on the vertices of $V(D) \backslash\{x, y\}$. Note that $D^{\prime}$ is a balanced bipartite digraph on $2 a^{\prime}$ vertices, where $a^{\prime}=a-1$. For the sake of contradiction, suppose that $l>2$, that is, $D^{\prime}$ is not hamiltonian. By (3.8) and $(3.9)$, we note that $\stackrel{\leftrightarrow}{a}\left(\{u\}, V\left(D^{\prime}\right)\right) \geq$ $2 a^{\prime}-1$ for all $u \in V\left(D^{\prime}\right)$, which implies that $d_{D^{\prime}}(u)+d_{D^{\prime}}(v) \geq 4 a^{\prime}-2$ for all $u, v \in V\left(D^{\prime}\right)$. Therefore, if $a^{\prime} \geq 3$, then $4 a^{\prime}-2 \geq 3 a^{\prime}+1$ and condition $\left(\mathcal{M}_{1}\right)$ (hence also $\left(\mathcal{M}_{0}\right)$ ) holds for $D^{\prime}$. Then, by induction, $D^{\prime}$ is hamiltonian; a contradiction. It follows that $a^{\prime} \leq 2$, which implies that $a^{\prime}=2, l=3$ and $\left|C_{1}\right|=\left|C_{2}\right|=\left|C_{3}\right|=2$. In this case, we have $4 a^{\prime}-2=3 a^{\prime}$, so condition $\left(\mathcal{M}_{0}\right)$ holds for $D^{\prime}$ which, by induction, implies that $D^{\prime}$ is not strongly connected. However, some vertex $u \in V_{2} \backslash V\left(C_{1}\right)$ must have $u x \notin A(D)$ or $x u \notin A(D)$, as otherwise $d(x)=2 a>a+1$, contrary to the assumption of Case 2a. For such $u$, by [3.9), we have $\stackrel{\leftrightarrow}{a}\left(\{u\}, V\left(D^{\prime}\right)\right)=2 a^{\prime}$. Therefore, there are arcs in both directions between $C_{2}$ and $C_{3}$ implying that $D^{\prime}$ is strongly connected; a contradiction. We thus proved that $l=2$.

Pick any $u \in V_{1} \backslash\{x\}$. Suppose that $u y \in A(D)$. Then $x u^{+} \notin A(D)$ (for else we could merge $C_{1}$ and $C_{2}$ ), and hence $u^{+} x \in A(D)$, by (3.9). Consequently, $y u^{++} \notin A(D)$ (where $u^{++}$is the successor of $u^{+}$on $C_{2}$ ), and hence $u^{++} y \in A(D)$, by (3.8). Repeatedly using this argument, we eventually arrive at $u^{-}$, the predecessor of $u$ on $C_{2}$ : Its predecessor $u^{--}$dominates $y$, so $x u^{-} \notin A(D)$. Then $u^{-} x \in A(D)$, by [3.9), and thus $y u \notin A(D)$. Therefore we obtain that $A\left[V\left(C_{1}\right), V\left(C_{2}\right)\right]=\varnothing$, which contradicts the strong connectedness of $D$.

If, in turn, $u y \notin A(D)$, then $y u \in A(D)$, by $(3.8)$, hence $u^{-} x \notin A(D)$ (for else we could merge $C_{1}$ and $C_{2}$ ), and so $x u^{-} \in A(D)$, by (3.9). Consequently, $u^{--} y \notin A(D)$, hence $y u^{--} \in A(D)$, by (3.8). Repeatedly using this argument, we get that $A\left[V\left(C_{2}\right), V\left(C_{1}\right)\right]=\varnothing$, which is impossible in a strongly connected $D$. This contradiction completes the proof of Case $2 \mathrm{a}$. 


\section{Case $2 b$.}

$d(x)<a+1$.

Then, by condition $\left(\mathcal{M}_{0}\right)$, we have $d\left(x^{\prime}\right) \geq 2 a$ for all $x^{\prime} \in V_{1} \backslash\{x\}$. Hence, in fact, $d\left(x^{\prime}\right)=2 a$ for all such $x^{\prime}$, and $d(x)=a$, by $\left(\mathcal{M}_{0}\right)$ again. Now, $A(D)$ contains all the arcs between the vertices of $V_{1} \backslash\{x\}$ and $V_{2}$. Also, $A(D)$ contains $x y, y x$, and at least one arc of the form $x y^{\prime}$ or $y^{\prime} x$ for some $y^{\prime} \in V_{2} \backslash\{y\}$ (because $d(x)=a \geq 3$ ). One readily sees that such a $D$ is necessarily hamiltonian. This contradicts the hypothesis that $l>1$, which completes the proof of the theorem.

\section{Acknowledgements}

We would like to thank the anonymous referee for many valuable suggestions that allowed us to improve the exposition of our results.

\section{References}

[1] J. Adamus and L. Adamus, A degree condition for cycles of maximum length in bipartite digraphs, Discrete Math. 312 (2012), 1117-1122.

[2] D. Amar and Y. Manoussakis, Cycles and paths of many lengths in bipartite digraphs, J. Combin. Theory Ser. B 50 (1990), 254-264.

[3] J. Bang-Jensen and G. Gutin, "Digraphs: Theory, Algorithms and Applications", 2nd edition, Springer, London, 2009.

[4] C. Berge, "Graphs and hypergraphs", North-Holland, Amsterdam, 1973.

[5] J.C. Bermond and C. Thomassen, Cycles in digraphs - a survey, J. Graph Theory 5 (1981), 1-43.

[6] J.A. Bondy and C. Thomassen, A short proof of Meyniel's theorem, Discrete Math. 19 (1977), 195197.

[7] A. Ghouila-Houri, Une condition suffisante d'existence d'un circuit hamiltonien, C. R. Acad. Sci. Paris 251 (1960), 495-497.

[8] Y. Manoussakis and I. Milis, A sufficient condition for maximum cycles in bipartite digraphs, Discrete Math. 207 (1999), 161-171.

[9] M. Meyniel, Une condition suffisante d'existence d'un circuit Hamiltonien dans un graphe orienté, J. Combinatorial Theory Ser. B 14 (1973), 137-147.

[10] C.St.J.A. Nash-Williams, Hamilton circuits in graphs and digraphs, in "The many facets of graph theory”, Springer, Lecture Notes 110 (1969), 237-243.

[11] D.R. Woodall, Sufficient conditions for circuits in graphs, Proc. London Math. Soc. 24 (1972), 739 755. 